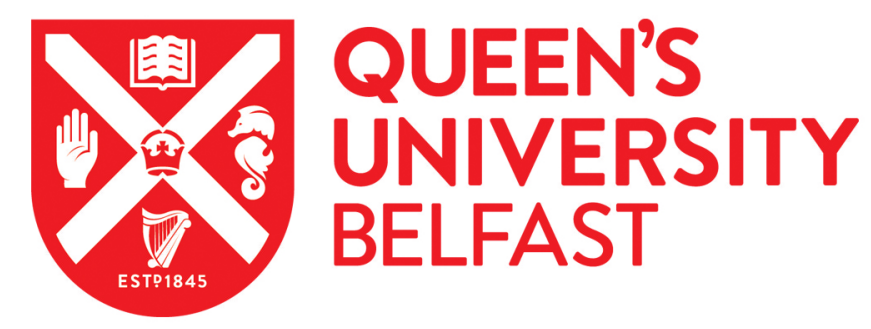

\title{
Family carers' experiences of participating in a weight management program for overweight children and adolescents with intellectual disabilities: An exploratory study
}

Lee, R. L. T., Brown, M., Leung, C., Chen, H., Louie, L., Chen, J. L., \& Lee, P. H. (2018). Family carers' experiences of participating in a weight management program for overweight children and adolescents with intellectual disabilities: An exploratory study. Journal of Advanced Nursing. https://doi.org/10.1111/jan.13845

Published in:

Journal of Advanced Nursing

Document Version:

Peer reviewed version

Queen's University Belfast - Research Portal:

Link to publication record in Queen's University Belfast Research Portal

Publisher rights

๑) 2018 John Wiley \& Sons Ltd. This work is made available online in accordance with the publisher's policies. Please refer to any applicable terms of use of the publisher.

\section{General rights}

Copyright for the publications made accessible via the Queen's University Belfast Research Portal is retained by the author(s) and / or other copyright owners and it is a condition of accessing these publications that users recognise and abide by the legal requirements associated with these rights.

Take down policy

The Research Portal is Queen's institutional repository that provides access to Queen's research output. Every effort has been made to ensure that content in the Research Portal does not infringe any person's rights, or applicable UK laws. If you discover content in the

Research Portal that you believe breaches copyright or violates any law, please contact openaccess@qub.ac.uk. 


\title{
Family carers' experiences of participating in a weight management program for overweight children and adolescents with intellectual disabilities: an exploratory study
}

\begin{abstract}
Aim: To gain insight into the experiences of family carers participating in a weight management program via mHealth tools for overweight children and adolescents with intellectual disabilities.
\end{abstract}

Background. Many weight reduction programs fail to show positive and sustainable impacts due to not involving parents, who are usually unavailable to attend school-based health programs. The mHealth interactive interventions were carried out in September 2015 August 2016, engaging carers to monitor and sustain their children's healthy lifestyle behaviors at home being one way to achieve this.

Design. Qualitative research design to conduct focus group discussion with family carers involved in a school-based weight management program via the mHealth interventions.

Methods. An exploratory study was used to examine family carers' views of participating in a weight management program. Focus group interviews examined the in-depth experiences of 20 family carers in providing social support and monitoring lifestyle behaviors via the mHealth interactive interventions.

Results. Twenty family carers were recruited, and four themes emerged: (1) improving family carer-child interactions and communications, (2) gaining useful and practical health information from experts, (3) supporting each other via an mHealth platform, and (4) appreciating the collaborative effort between school personnel and family carers. Family carers stressed the usefulness of the mHealth interactive interventions in monitoring and sustaining the children's healthy lifestyle behaviors at home.

Conclusion. The mHealth interactive interventions for increasing family carers' involvement and monitoring were well received. The findings provide new insights into using mHealth interventions in future weight management programs involving parental participation in the home environment.

Keywords: intellectual disabilities, overweight children and adolescents, family carers' participation, mHealth interactive interventions, weight management program involving parental participation 


\section{Introduction}

There is a high prevalence of obesity among children with disability and special needs. The prevalence of childhood overweight and obesity in children aged 5-19 years old was $6.2 \%$ and $13.8 \%$ respectively ( $\mathrm{Li}$ et al., 2010). It is increasingly recognized as an international phenomenon and one that requires active intervention (Centers for Disease Control and Prevention: Disability and Obesity, 2017). Overweight children and adolescents with intellectual disability may not have a sufficient understanding of obesity or its related comorbidities. For this reason, many children with disability will grow into obese adults with all the associated chronic health problems, such as diabetes, back and joint pain, cardiovascular disease and hypertension (Gibbs et al., 2008). The Centers for Disease Control and Prevention report that children with disability are $38 \%$ more likely to be obese than other children. Children with disabilities are at higher risk of obesity due to their lower engagement in physical activity than children of the same ages without disabilities (Murphy, Carbone \& American Academy of Pediatrics Council on Children with Disabilities, 2008).

Previous studies have only focused on short-term and individual outcome measures in weight management programs for children with disabilities, without involving parental participation (Speiser et al., 2005, Tong et al., 2012). There is still little information provided on the potential adoption or sustainability of weight management programs for this group (Anderson \& Heyne, 2010, Hogan, McLellan, \& Bauman, 2000).

Thus, a weight reduction program requires effective interventions targeting the social forces and collective efficacy of these health-related behaviors in overweight or obese children and adolescents with mild intellectual disability (MID) in a school setting. Diet, physical activity and sedentary behaviors are predicted by a set of factors from multiple domains (Glanz \& Bishop, 2010). It is therefore important to study not only how lifestyle behaviors relate to 
weight status, but also the social forces and collective efficacy that influence and shape these behaviors. Children and adolescents with disabilities have many characteristics beyond those of typically developing children that increase their risk for greater body mass due to excess use of medication. Symptoms related to obesity are those such as joint pain, having different dietary habits related to their disability, such as a fluid diet, as well as requiring support and supervision because of their cognitive impairment in participating and engaging in physical activity and weight reduction strategies (Grondhuis \& Aman, 2013). Given the central role of behavioral intervention, much more research is needed in this area.

\section{Background}

While childhood obesity has been the focus of considerable research in recent years, longerterm follow up is needed to confirm the maintenance of treatment effects for all types of interventions. Interventions that work for typically developing children may not work for obese children with disabilities without modification, and those that do work may not be available in their community. These interventions are more likely to be effective when implemented during childhood, when individuals are still growing and forming life-long health behaviors (Lazaar et al., 2007). Both school and home can provide a supportive physical and social environment for preventing childhood obesity. It is important to develop an evidence-based program, such as mHealth, that facilitates the parent's participation in weight loss maintenance interventions and thus can have a positive impact on a large proportion of the target population. These interventions have the potential to positively impact the individual, their family and wider care services. Thus, a structured, school-based weight management intervention is needed to improve health outcomes.

There is clear evidence of the efficacy of interventions to establish health-promoting or health-enhancing behaviors at an individual level to reduce health-risk behaviors such as 
obesity and the wider associated health complications (Lee et al., 2017a). However, these behavior changes are frequently difficult to maintain, especially for children with intellectual disability, which poses an important challenge in planning weight loss maintenance programs for this population.

Few studies have focused on family carers' perspectives on behavioral change by engaging their participation in a school-based weight management program (SBWMP) via mHealth tools for the overweight children and adolescents with intellectual disability.

The limited maintenance of behavioral change seen in initial intervention efforts may be due to the failure to consider contextual factors such as collective efficacy, which emphasizes interaction and observational learning on a group level. Collective efficacy (social control and social cohesion) is a concept used in social cognitive theory to describe a group's ability to achieve goals (Bandura, 2000); it is related to the social environment, which predicates community collective efficacy via socialization with social forces and a social network, as shown by Kawachi and Berkman (2001). Thus, further research should aim to achieve greater integration of social constructs in behavioral theories and interventions as social factors that can affect and sustain change. The current study aimed to explore family carers' experiences after participating in a 12-month SBWMP via mHealth tools with interactive intervention.

\section{mHealth intervention protocol}

The mHealth intervention protocol provides guidelines and examples for implementing strategies and methods used in the extended home environment of a school-based weight management program for overweight children and adolescents with mild intellectual disability (Figure 1). The cognitive impairment of students with intellectual disability results in poor self-regulation, planning, execution and monitoring of their lifestyle behaviors, with the consequences of increased prevalence of chronic illnesses among this vulnerable 
group. Adopting the principles of collective efficacy theory, we examined whether a groupmediated school-based weight management intervention, extended to the home environment and involving the family caregivers, for weight loss among overweight children and adolescents with mild intellectual disability resulted in changes in self-regulatory selfefficacy for healthy eating behaviors and practicing physical activity, and whether these changes mediated weight loss. Details of the 12-month school-based weight management program extended to the home environment involving family carers' participation via mHealth tools are given in Table 2. In this study, family carers referred to parents (mothers and fathers) and caregivers, extended and chosen families who contribute significantly to the wellbeing of children or young people and who may or may not have legal responsibility.

\title{
The study
}

\begin{abstract}
Aim
The aim of this study was to gain an in-depth understanding of family carers' experiences of participating in a weight-management program via an mHealth interactive intervention for the overweight children and adolescents with MID. This understanding is required to guide the formation of successful structured weight-management interventions for this vulnerable group.
\end{abstract}

\section{Design}

An exploratory qualitative study was used to examine family carers' experiences of participating in a structure weight management program for the overweight children and adolescents via mHealth intervention.

\section{Participants}


A purposive sample of family carers were recruited from special schools for overweight children and adolescents with MID. The participants were informed of the aim of the study. The inclusion criteria were: (a) family carer understands and speaks Cantonese, (b) family carer participates in the mHealth intervention activities, (c) family carer has a child or an adolescent aged 8-16 with MID based on a standardized intelligence test (IQ score of 50-69) who are studying in P.3-F.4 in special schools in Hong Kong, (d) family carer has a child or an adolescent free from physical impairment and cardiovascular disease, based on their school medical record, (e) family carer is able to follow the instructions and understand the teaching materials as posted by the school nurse and research team members via mHealth tools, and (f) family carer has a child or an adolescent whose BMI-for-age is in the overweight ( 85 th to $<95$ th percentile) or obese ( $>$ or $=95$ th percentile) categories. In contrast, the study excluded family carers of a child or adolescent aged over 16 whose BMI-for-age was between the $85^{\text {th }}$ and $95^{\text {th }}$ percentiles and who had moderate intellectual disabilities. Family carers were contacted either through the school communication system or by telephone. If they were interested, they then received an invitation letter with a consent form and an information sheet detailing the aim of the study. There were forty-three family carers participated in the SBWMP via mHealth intervention. Twenty family carers comprised parents, grandparents and auntie from four special schools were invited to participate in this study, and their characteristics were as shown in Table 1.

\section{Data collection}

Focus group discussions were conducted between January and April 2017 by the principal investigator and the research team members in the schools' meeting rooms. Consents were obtained prior to conducting the focus group discussions. The discussion guide was derived from the intervention (Appendix I). There were five focus group discussions, each consisting 
of four family carers, who engaged by providing support to reinforce and monitor the taught healthy lifestyle behaviors for the overweight children and adolescents with MID at home. Each focus group discussion lasted 45-60 minutes and started with the same question: 'Tell us your experience of participating in your child's weight management program via mHealth interactive activities'. Family carers were encouraged to share their stories in a friendly atmosphere in the school's meeting room. Each of the five focus group interviews had the full participation of four family carers.

\section{Ethical considerations}

Ethical approval was obtained from the University Research Ethical Committee prior to recruiting the family carers. The purpose of the study was explained to them, and their informed consents were obtained regarding their voluntary participation. Permission to audiorecord the interviews was obtained. The family carers' personal information was not disclosed. The collected data were only accessible to the research team. Confidentiality was ensured, with full respect for the anonymity of the family carers.

\section{Data analysis}

All the focus group discussion data were tape-recorded and transcribed into Cantonese. The audiotapes were then listened to a second time to ensure that the transcript of each interview was complete and accurate. Data were analyzed using thematic analysis (Braun \& Clarke, 2006). This was a systematic procedure whereby verbatim or unstructured data were systematically coded by content area. The principal investigator and two other research team members analyzed the data independently, in Cantonese, in the first phase of data analysis. All the data was read line-by-line first. The team then shared and discussed the initial and refined codes after review by each individual in the second phase. The meaningful 
expressions from the family carers were then identified, extracted as units and grouped into initial codes and refined codes in the coding tree and finally immersed into distinctive subthemes and themes in the final phase as shown in Figure 2.

\section{Reflexivity}

The reporting guidelines of the COREQ were adopted in this study, with three domains: 1) research team and reflexivity, 2) study design, and 3) analysis and findings (Tong, Sainsbury, \& Craig, 2007). The COREQ aims to improve the transparency of all aspects of qualitative research by providing clear reporting standards. It is a 34-item checklist to help authors report important aspects of the study methods, findings, analysis and interpretations (Tong, Sainsbury, \& Craig, 2007).

The research assistants and principal investigator (PI) conducted five focus group discussions with family carers to examine their experiences of participating in an mHealth intervention. The PI was a qualitative researcher for over 15 years, thus she has the passion and commitment to conduct qualitative research studies to examine participants' perspectives. For this study, she investigated the in-depth experiences of family carers after involvement in the mHealth intervention. The PI recognizes that qualitative research is particularly beneficial in revealing practices that are taken for granted and the meanings embedded in everyday experiences. Thus, she recognizes that it is important to construct reality according to interpretations of the data regarding participants' experiences.

It was used when the focus group questions asked for the participants' experiences and the PI did not bracket her biases and prior engagement with the question under study. The research was reflected upon, on an ongoing basis, to allow researchers to provide considerable thought 
to their own experience, and to clearly explain the ways in which participants found meaning in and related to the issues being researched (Fossey et al., 2002).

For this study, the world of Chinese family carers of children and adolescents with intellectual disability could be expanded by helping carers to see new roles and commitment in promoting healthy lifestyle behaviors in a home setting. Using a qualitative study design, the PI believed that the carers' world could be expanded only in relation to existing concerns and possibilities by understanding their participation experiences in monitoring and sustaining healthy behaviors for the children and adolescents with intellectual disability in the home environment. This understanding allowed further exploration and revelation of the underlying meanings of these carers' experiences, namely the impact of mHealth on carers' experiences. Therefore, the experience and role of these family carers of overweight children and adolescents with intellectual disability could be elaborated via focus group discussions. In addition, the research delved into the relationships and meanings that knowledge and context have for each other.

Only the first two focus group discussions were conducted to adjust the pre-developed discussion guide (Appendix I). The researcher establish relationships of trust, observation and referential adequacy, and through prolonged engagement with the family carers. Five focus group discussion data were validated by another researcher for peer validation purposes. Data quality for bias and coding checks were made by two researchers and one nurse clinician to establish dependability.

\section{Findings}

Twenty participants, 4 males and 16 females, with ages ranging from 25-65, participated in five focus group discussions (Table 1). They were 16 parents, three grandparents and one 
aunt recruited from the participants of weight management program via mHealth intervention. Each focus group discussion was conducted in Chinese in the school setting and lasted between 45 and 60 minutes. The focus group discussion guide was derived from the family carers' experiences in a SBWMP extended to the home environment via interactive mHealth tools (Table 2).

In this study, the family carers stated that the participation of mHealth weight management program had motivated them to learn relevant health information how to promote children and adolescents to practice healthy lifestyle behaviors. It also evoked a feeling of shared experience with other family carers via mHelath tools. Thereafter, four main themes with eight subthemes emerged from the data analysis. The four main themes were (1) improving carer-child interactions and communications, (2) gaining useful and practical health information from experts via mHealth tools, (3) supporting each other via the mHealth platform, and (4) appreciating the collaborative effort between school personnel and family carers. The eight subthemes were i) enhance participation, ii) engage in activities between children and their parents, iii) interact with professional groups, iv) obtain update and relevant health knowledge, v) motivate each other, vi) advocate healthy child development vii) establish communication platform between school and home, and viii) appreciate continuity of care to foster child health. Family carers stressed the practicality of interventions in the mHealth SBWMP in terms of facilitating their involvement in learning, monitoring and sustaining their children's healthy lifestyle behaviors at home, although they sometimes found the information a bit overwhelming with all the instant messages via the mHealth tools. Family carers' participation in SBWMP via mHealth tools enhances their health knowledge and their support for the overweight children and adolescents with intellectual disability in the home setting. This is essential in enabling them to be good models and supporters, influencing and sustaining the children's and adolescents' healthy lifestyle behaviors such as 
healthy dietary habits, practicing regular physical activity, having positive energy and becoming positive. The use of mHealth tools to encourage family carers' participation in sustaining weight loss interventions for the overweight children and adolescents with intellectual disability is feasible and appropriate. It involves three levels of participationindividual, school and family-to promote child health in the community.

\section{Theme 1: Improving family carer-child interactions and communications}

The communication between family carers and children was not good prior to participating in the weight management program. Family carers reported a positive impact of the program on family members' relationships, especially those with the child. They also said that interactions with the child had increased as they reinforced and monitored the health information posted using the interactive mHealth tools. The two subthemes are to i) enhance participation and ii) engage in activities between children and their parents.

'I participated more often in outdoor activities and public events with my son after joining this program. This has improved our family relationship.' (Participant No. 16)

Most of the family carers were aware of the benefits to students of practicing healthy lifestyle behaviors. Thus, some of them were willing to do exercises with the students to show their support and provide encouragement. They also stated that by so doing, their interactions and communications with the students had improved. They also expressed that the overall atmosphere in the home environment had improved after joining the program.

'(He) became more energetic after participating in the weight management program extended to home via the mHealth interactive intervention. The program inspired him to dance freely to relax his mind. He now loves dancing; he's always dancing while watching 
TV ... He also likes going for a walk and coming with me to the market.' (Participant No.

As the mHealth interactive activities involved the parents, they reported that the overweight children and adolescents with intellectual disability had become more active and sociable. After participating in the intervention, their intention and willingness to exercise increased. They expressed that their children enjoyed exercising with their peers and parents.

'After participating in the program at school, I observed that he gradually changed his dietary habits by eating more fruits and vegetables. He also exercises more regularly, such as by swimming and hiking. Even when I am not at home, my older son helps me to get my son to exercise, e.g. doing step-ups. We also participated as a family in a marathon that was held by the school.' (Participant No. 1)

\section{Theme 2: Gaining useful and practical health information from experts via mHealth tools}

Family carers reported that they were aware of the benefits of participation in the schoolbased weight management intervention via mHealth interactive tools, as they were very busy at work every day and came home late. They admired the multidisciplinary experts' effort involved in facilitating the engagement of the carers with the school weight management program.

'The WhatsApp group is certainly very helpful in enabling us (the parents) to learn about nutrition and to improve the quality of our family diet.' (Participant No.1)

All the family carers acknowledged that the mHealth tools empowered them to reinforce and monitor their child's health behaviors by providing relevant health information. Through the interactive intervention via mHealth tools, they knew more about the factors and barriers to practicing healthy lifestyle behaviors, such as the benefits of regular exercise to keep the 
body healthier, reading food labels to decrease fat, salt and sugar intake, and tips for preparing healthier food. Thus, the first subtheme was to interact with professional groups. The instructions helped them to adjust their quality of life for the overweight children and adolescent with intellectual disability and their family. 'Through the WhatsApp group, parents can find out what children learned in physical activity at school, like dancing, and practice with them at home.' (Participant No.8)

'On the other hand, the SBWMP via mHealth tools also increased our knowledge of the benefits of regular exercise as highlighted by the experts in the WhatsApp group. We now constantly remind our son to exercise daily. I act as a role model by going for a walk with him.' (Participant No.16)

Participants reported that the mHealth tools motivated them in supporting and monitoring their overweight and obese children with MID. The improvement in their physical condition, such as weight loss and weight maintenance, the increased knowledge about health, and the positive changed behavior of the children motivated the participants to undertake further monitoring.

'My daughter was more aware of her body condition. She took the initiative of managing her body weight by weighing herself from time to time, running on treadmills at home and eating less at school. When she noticed any weight gain, she would do more exercise to reduce it. The positive impacts on her motivated me to monitor her lifestyle.' (Participant No. 8)

In addition, monitoring the health condition of the overweight and obese children with MID was facilitated by the mHealth tools. It acted as a platform in helping the participants to understand what the program provided to the child. Thus, the second subtheme was to obtain update and relevant health knowledge. 
'I could keep up with what my kid was learning in the program. This helped me to manage his daily life. ... Through the 'WhatsApp' group, I came to understand the importance of exercise. Our family bought a treadmill so that he could run whenever he wanted and whatever the weather.' (Participant No.4)

\section{Theme 3: Supporting each other via the mHealth platform}

The family carers reported that they were more willing to act as role models and to teach and follow up on their child's lifestyle behaviors by integrating health information into their daily living. Few family carers said that they learnt and shared information related to supporting and monitoring the children's and adolescents' lifestyle behaviors and support to each other via mHealth tools.

'The discussion in the WhatsApp group via the mHealth platform has provided us a platform to learn and support each other, and I feel like there is always someone to talk to when I need it." (Participant No. 13)

The family carers indicated that the mHealth tools were like a hub that provided useful and practical information about healthy lifestyles from healthcare professionals, and shared experience and gave mutual support to other family carers. Therefore, an atmosphere of encouraging and reminding each other was promoted. The two subthemes are to motivate each other and to advocate for healthy child development.

'The WhatsApp group is certainly very helpful in enabling [parents] to learn about ingredients in certain nutritional food and to improve the quality of our family diet. Before, we were not as aware of nutrition information or the nutritional content of the food we consumed. We as parents can promote and support each other via mHealth tools even though we have never met each other before'. (Participant No. 1) 
Some of the family carers expressed that they found it difficult to engage their children with intellectual disability in the program due to their reduced attention span. These carers suggested that more games and animation be included, rather than strictly information.

\section{Theme 4: Appreciating the collaborative effort between the school personnel and the family carers}

With all the health information available via the mHealth tools, family carers became more knowledgeable on how to reinforce and monitor their children's healthy lifestyle behaviors. They became more willing to encourage their children to practice healthy dietary habits and exercise regularly at home, compared with when their child only participated in the program at school. The two subthemes are to establish communication platforms between the school and home and appreciate continuity of care to foster child health.

'He likes to eat vegetables and my parents are monitoring the amount of meat he eats. My mum cooks more vegetables and less meat than before, so the quality of my diet has also improved somewhat. For example, I only eat one chicken wing, because I know they are rather fattening.' (Participant No. 3)

The working hours of some of the family carers were too long, so it was difficult for them to participate in the children's school activities. The mHealth interactive intervention provided opportunities for them to get involved in the children's weight management program at school. This facilitated the transfer of the children's sustainable healthy lifestyle behaviors from school to the home environment with the family members' reinforcement and monitoring.

'The information on how to read food labels via WhatsApp is useful and practical. I follow the food pyramid when preparing meals. It would be lovely if the WhatsApp group kept updating the health information. Children tend to follow your advice. This 
also demonstrates the collaboration between the school and families.' (Participant No. 5)

\section{Discussion}

The findings of this study reflect the impact of the combined efforts of the school personnel and family carers. These findings are consistent with those of the Norwegian School of Sport Sciences, suggesting that an intervention effect on targeted determinants would mediate changes in weight status with parental or caregivers involvement in children's physical activity (Bergh, 2012). The family carers in this study also reported that the mHealth interactive interventions had facilitated their motivation to reinforce and monitor the children's lifestyle behaviors at home. Some of the family carers expressed that the WhatsApp group was certainly very helpful to motivate them to learn about the new health information and to enhance their role in monitoring and supporting the children and adolescents to practice healthy lifestyle behaviors. It is important to enhance the effective communications and collaboration between school and family via advance technology such as mobile phone tools to provide health knowledge for partnership of care. Robinson and his colleagues (2013) reported that combining interactive activities via mHealth tools is superior to conventional interventions that do not involve the family members of overweight children with intellectual disability.

The results of this qualitative study reflected teachers' and family carers' perceptions of feasibility, benefits and limitation of SBHHP in the longer-term follow-up of weight loss maintenance involving carers through interactive multimedia systems and advanced technology.

This qualitative study result will also guide school nurses in the development of a structured school-based weight reduction program using mHealth tools to interact with family 
carers (Lee et al., 2017b). Dishman and his colleagues (2005) stated that a weight management program combined with interactive games that engage parents' participation via mHealth tools is innovative and cost-effective. A school nurse or paediatric nurse does have an essential role to bridge the gap between schools and families and enhance communication.

The use of mHealth tools to encourage family carers' participation in sustaining weight loss interventions for overweight children and adolescents with intellectual disability is feasible and appropriate. It involves three levels of participation in the school community-individual, school and family - to promote child health. This finding is shaped in part by Bronfenbrenner's Ecological Systems Theory (1986) which consists of five environmental systems, which are microsystem, mesosystem, exosystem, macrosystem and chronosystem. Each layer interacts with each other. According to the Bronfenbrenner's ecological systems theory (1986), it is important not only to examine the individual child and his or her immediate environment which is the family and peers, but also assessing the interactions between the larger environments such as the school and community, which can have influences on the child's development.

The findings of this study support that the influence of attitudes and behaviors within parentteacher interactions in a reciprocal partnership which represents a promising avenue that the school nurse has an essential role to bridge this achievement. This reciprocal partnership is also supported by Deslandes (2001) who described this partnership as 'A bridge to the futurecollaboration between parents, schools and communities.'

\section{Limitations}

The first limitation of this study is that the family carers were recruited from direct contacts at institutions for mildly intellectually disabled students. This makes it difficult to generalize the 
findings to all children with special needs. A second limitation is the effective translation of trials to real-world settings. Generalization of effective interventions requires an expansion of the assessment of intervention outcomes delivered in diverse settings. Community- and organization-wide interventions have had varied success. The findings are marred by poor designs and methods. In general, however, interventions that are more broadly based and multifaceted are more likely to be effective. These challenges are not confined to advances in individual behavior change, as similar challenges apply to all levels of intervention.

\section{Conclusion}

It is crucial to involve the family carers' and assess their perceptions and experiences in evaluating the impact of an intervention targeting outcome measures. Overweight children and adolescents with intellectual disability in a special school learn to behave through constructs of both instruction (i.e. how parents, teachers, and other authorities and role models tell them to behave) and observation (i.e. how they see adults and peers behaving). Family carers' participation via a mHealth interactive intervention enhances their health knowledge and their support for overweight children and adolescents with intellectual disability in the home setting. The findings of this study have provided new insight into the assistance that the mHealth intervention can offer school nurses in developing and planning weight management programs that promote child health in the community. New technology like the mHealth intervention allows parents and caregivers to enhance the health and wellbeing of their children, resulting in better health outcomes. These essential elements in the school health promotion programs are valued by the parents, as they have participated and remain involved. It is essential to be good role models and supporters in the home environment, influencing and sustaining participants' healthy lifestyle behaviors such as healthy dietary habits, practicing regular physical activity, having positive energy and 
becoming positive.

An essential element in the planning of behavioral change health programs to facilitate the efficacy of interventions to establish health-promoting or health-enhancing behaviors such as diet and physical activity, and to reduce health-risk behaviors such as obesity and the wider associated health complications. However, these behavior changes are frequently difficult to maintain, which poses an important challenge to the field. The limited maintenance of behavioral change seen in initial intervention efforts may be due to the failure to consider all these contextual factors, such as social support and social networks via socialization (Hatch et al., 2013). Thus, further research should target greater integration of social constructs in behavioral theories and interventions as social factors that can affect and sustain change by involving parents in collaboration with health care professionals such as school nurses to promote children's health in school communities.

\section{Acknowledgements}

The authors would like to express their sincere gratitude to the special schools that participated in this study.

\section{Funding}

This project was funded by the General Research Fund 2014/2015 (No. PolyU 15402714).

\section{Conflict of interest}

No conflict of interest has been declared by the author(s).

\section{Authors' contributions}


Study design: RL, MB; data collection: RL, PL; data analysis: RL, CL; manuscript preparation: RL, CY, MB, PL.

\section{References}

Anderson, L., \& Heyne L. (2010). Physical activity for children and adults with disabilities: an issue of 'amplified' importance. Disability of Health Journal, 3(2), 71-73.

Bandura, A. (1982). Self Efficacy Mechanism in Human Agency. American Psychologist, $37(2), 122-147$.

Bandura, A. (1987). Self-efficacy: Toward a unifying theory of behavior change. Psychological Review, 84(2), 191-215.

Bandura, A. (2000). Exercise of human agency through collective efficacy. Current Directions in Psychological Science, 9(3), 75-78.

Bergh, I.H., Bjelland, M., Grydeland, M., Lien, N., Andersen, L.F., Klepp, K.I., ... Ommundsen Y. (2012). Mid-way and post-intervention effects on potential determinants of physical activity and sedentary behavior, results of the HEIA study - a multicomponent school-based randomized trial. The International Journal of Behavioral Nutrition and Physical Activity. 9(63). https://doi.org/10.1186/1479-5868-9-63.

Braun, V., \& Clarke, V. (2006,) Using thematic analysis in psychology. Qualitative Research in Psychology, 3(2), 77-101.

Bronfenbrenner, U. (1986). Ecology of the family as a context for human development: Research perspectives. Development Psychology, 22, 723-742.

Centres for Disease Control and Prevention. (2017). Disability and Health Data System. Atlanta: Centers for Disease Control and Prevention.

Deslandes, R. (2001). A vision of home-school partnership: three complementary conceptual Frameworks. In the proceedings ed. F. Smit, K. van der Wolf \& P. Sleegers. ITS Stichting Katholieke Universiteit to Nijmegen NL.

Dishman, R.K., Motl, R.W., Saunders, R., Felton, G., Ward, D.S., Dowda, M., \& Pate, R.R. (2005). Enjoyment mediates effects of a school-based physical-activity intervention. Medicine and Science in Sports and Exercise, 37(3), 478-487.

Fossey, E., Harvey, C., McDermott, F., \& Davidson, L. (2002). Understanding and evaluating qualitative research. Australia and New Zealand Journal of Psychiatry, 36(6), 717-732.

Gibbs, S.M., Brown, M.J., \& Muir, W.J. (2008). The experiences of adults with intellectual disabilities and their carers in general hospitals: a focus group study. Journal of Intellectual Disability Research, 52(12), 1061-1077.

Glanz, K., \& Bishop, D.B. (2010). The role of behavioral science theory in the development and implementation of public health interventions. Annual Review of Public Health, 31, 399-418.

Grondhuis, S.N., \& Aman, M.G. (2013), Overweight and obesity in youth with developmental disabilities: a call to action. Journal of Intellectual Disability Research, 58(9), 787-799.

Hatch, S.L., Harvey, S.B., Dandeker, C., Burdett, H., Greenberg, N., Fear, N.T., \& Wessely, S. (2013), Life in and after the Armed Forces: social networks and mental health in the UK military. Sociology of Health \& Illness, 35(7), 1045-1064.

Hogan, A., McLellan, L., \& Bauman, A. (2000), Health promotion needs of young people with disabilities: a population study. Disability \& Rehabilitation, 22(8), 352-357. 
Kawachi, I., \& Berkman, L.F. (2001). Social ties and mental health. Journal of Urban Health: Bulletin of the New York Academy of Medicine. 78(3), 458-467.

Lazaar. N., Aucouturier, J., Ratel,S., Rance, M., Meyer. M., \& Duché, P. (2007). Effect of physical activity intervention on body composition in young children: influence of body mass index status and gender. Acta Paediatrica, 96(9), 1321-1325.

Lee, R.L., Lee, P.H., Sze, D.M., \& Chien,W.T. (2017a). Anthropometric profile of Hong Kong children and adolescents: The wellness population of youth study. Journal of the American Society of Hypertension, 11(4), 196-203.

Lee, R.L., Leung, C., Chen, H., Louie, L., Brown, M., Chen, J.L., .. \& Lee P.H. (2017b). The impact of a school-based weight management program involving parents via mHealth for overweight and obese children and adolescents with intellectual disability. Journal of Environmental Research and Public Health, 14(10), 1178.

Li, Y., Hu, X., Zhang, Q., Liu, A., Fang, H., Hao, L., .. \& Ma G. (2010). The nutrition-based comprehensive intervention study on childhood obesity in China (NISCOC): a randomized cluster-controlled trial. BMC Public Health, 10, 229.

Murphy, N.A., Carbone, P.S., \& American Academy of Pediatrics Council on Children with Disabilities. (2008). Promoting the participation of children with disabilities in sports, recreation, and physical activities. Pediatrics, 121(5), 1057-1061.

Robinson, T.N., Killen, J.D., Kraemer, H.C., Wilson, D.M., Haskell, W.L., Pruitt, L.A., ... \& Varady, A. (2003). Dance and reducing television viewing to prevent weight gain in African-American girls: The Stanford GEMS pilot study. Ethnicity Disease, 13, S65-77.

Speiser, P.W., Rudolf, M.C., Anhalt, H., Camacho-Hubner, C., Chiarelli, F., Eliakim, A., ... \& Hochberg, Z. (2005). Consensus development: childhood obesity. Journal of Clinical Endocrinology and Metabolism, 90(3), 1871-1887.

Tong, A., Jones, J., Craig, J.C., \& Singh-Grewal, D. (2012). Children's experiences of living with juvenile idiopathic arthritis: A thematic synthesis of qualitative studies. Arthritis Care \& Research, 64(9), 1392-1404.

Tong, A., Sainsbury, P., \& Craig, J. (2007). Consolidated criteria for reporting qualitative research (COREQ): a 32-item checklist for interviews and focus groups. International Journal for Quality in Health Care, 19(6), 349-357. 